\title{
Heterochronic evolution reveals modular timing changes in budding yeast transcriptomes
}

Daniel F Simola ${ }^{1}$, Chantal Francis ${ }^{1}$, Paul D Sniegowski ${ }^{1}$, Junhyong Kim ${ }^{1,2^{*}}$

\begin{abstract}
Background: Gene expression is a dynamic trait, and the evolution of gene regulation can dramatically alter the timing of gene expression without greatly affecting mean expression levels. Moreover, modules of co-regulated genes may exhibit coordinated shifts in expression timing patterns during evolutionary divergence. Here, we examined transcriptome evolution in the dynamical context of the budding yeast cell-division cycle, to investigate the extent of divergence in expression timing and the regulatory architecture underlying timing evolution.

Results: Using a custom microarray platform, we obtained 378 measurements for 6,263 genes over 18 timepoints of the cell-division cycle in nine strains of S. cerevisiae and one strain of S. paradoxus. Most genes show significant divergence in expression dynamics at all scales of transcriptome organization, suggesting broad potential for timing changes. A model test comparing expression level evolution versus timing evolution revealed a better fit with timing evolution for $82 \%$ of genes. Analysis of shared patterns of timing evolution suggests the existence of seven dynamically-autonomous modules, each of which shows coherent evolutionary timing changes. Analysis of transcription factors associated with these gene modules suggests a modular pleiotropic source of divergence in expression timing.

Conclusions: We propose that transcriptome evolution may generally entail changes in timing (heterochrony) rather than changes in levels (heterometry) of expression. Evolution of gene expression dynamics may involve modular changes in timing control mediated by module-specific transcription factors. We hypothesize that genome-wide gene regulation may utilize a general architecture comprised of multiple semi-autonomous event timelines, whose superposition could produce combinatorial complexity in timing control patterns.
\end{abstract}

\section{Background}

Recent evolutionary studies using natural and inbred Drosophila and C. elegans lines have shown that genome-wide gene expression levels are much more conserved in nature than expected compared to independent measurements of mutational input [1-3], supporting the hypothesis that transcriptome evolution is characterized by stabilizing selection. These observations suggest that organisms show limited evolutionary divergence in gene expression via changes in gene regulation, either by qualitative changes in the connectivity of regulatory interactions or by quantitative changes in the strength of regulatory interactions. In addition, since the architecture of gene regulation involves highly

\footnotetext{
* Correspondence: junhyong@sas.upenn.edu

'Department of Biology, University of Pennsylvania, 433 S. University Ave., Philadelphia, PA 19104, USA

Full list of author information is available at the end of the article
}

connected and hierarchical cascades of control [4-7], regulatory change may be limited due to the broad potential for negative pleiotropic consequences [8]. Given this evidence for deleterious changes in gene regulation, how do organisms acquire transcriptome divergence?

Many studies have addressed this question by investigating the relationship between gene expression divergence and different kinds of genomic variation. Studies focusing on the regulatory effects of single nucleotide mutations have revealed that expression divergence generally associates with cis variation within species [9-13] and with trans variation between species [14-18]. Other studies have focused on larger, structural mutations, such as mobile element transposition or non-homologous recombination [19-21]. While these studies have discovered many important links between genomic variation and expression divergence, few studies have 
directly observed how genomic variation affects the qualitative structure or quantitative dynamics of an organism's genome-wide regulatory network. Notably, genome-wide binding patterns of six transcription factors were recently compared between two Drosophila species during embryonic development [22], revealing a dominant signature of quantitative, rather than qualitative changes in TF-DNA regulatory interactions.

One possible avenue for transcriptome divergence that remains consistent with the evidence of stabilizing selection on genome-wide gene expression levels and evolutionary conservation of gene regulatory network topology is that divergence might occur via changes in the timing of gene expression. Gene expression is both a quantitative trait and a dynamic trait, such that the timing of gene expression is regulated by a complex, polygenic combination of factors [5,23-26]. Evolutionary modifications to gene regulation have the potential to dramatically alter gene expression timing without greatly affecting mean expression levels [27,28]. Moreover, changes in the timing of regulatory factor expression could induce temporal shifts in the expression trajectories of some genes relative to others (heterochrony) $[29,30]$ without disrupting functional relationships.

In this study, we investigated the evolution of genome-wide gene expression as a dynamical system, to evaluate the pattern of divergence in expression timing, the mode of time-dependent transcriptome evolution, and the genome-wide architecture of timing control. We performed a large number of analyses and experiments that follow multiple inference pathways, as diagrammed in Figure S1 in Additional file 1. To overview our results and conclusions, we propose that our data and analyses support the following hypotheses: (1) while the vast majority of genes have bounded expression levels consistent with stabilizing selection, most expression trajectories show significant heterochronic divergence among strains; (2) the pattern of transcriptome divergence involves time-dependent changes in the magnitude, direction, and degrees of freedom of among-strain covariation; (3) genome-wide gene regulation utilizes a general architecture for transcriptome timing control comprised of distinct, coherent, and dynamically-autonomous modules; (4) population-level transcriptome divergence may predominantly result from quantitative changes in the expression dynamics of module-specific trans-regulatory factors rather than qualitative changes in the structure of genome-wide gene regulation; (5) an architecture involving modular timing control could generate complex patterns of heterochronic divergence combinatorially, while alleviating global negative pleiotropic effects associated with changes in regulatory interactions or changes in the expression of trans-regulatory factors.

\section{Results}

We assayed genome-wide gene expression (transcriptome) levels throughout the mitotic cell-division cycle (CDC) of ten natural budding yeast lines, including eight woodland and one laboratory strain of S. cerevisiae and one outgroup of $S$. paradoxus, in a comparative experimental design that involves technical, but not biological replicates of each timepoint (see Materials and methods). To calibrate the variation in gene expression across these lines with an expectation from mutationdrift, we also measured transcriptomes for 23 mutation accumulation (MA) lines. Normalizing and processing our data yielded expression levels for 6,263 genes at 18 sampled CDC-timepoints for the natural lines and unsynchronized expression for the MA lines. We validated our array measurements by comparison with previously published CDC-dependent temporal expression data (Figure S32 in Additional file 1) and with RNA sequencing data produced using the ABI SOLiD 3 platform (Figure S33 in Additional file 1). Our expression data show significant consistency both with previous CDC expression data and with quantification of RNA sequencing data.

\section{Genome-wide expression levels show much less variability than expected, but CDC-temporal expression patterns display broad divergence}

To assess the natural variability in genome-wide gene expression levels, we computed $F$-statistics at each timepoint $t$ for 4,973 genes $g$ exhibiting significant mutational variance [2] (see Supplemental materials and methods in Additional file 1). Each $F$-statistic is defined as the ratio of natural $\left(V_{n}\right)$ to mutational $\left(V_{m}\right)$ variances within $S$. cerevisiae, scaled by the divergence times of the natural and MA lines (in generations) and degrees of freedom: $F(g, t)=\frac{V_{n}(g, t)}{V_{m}(g)} \times \frac{600}{8.34 \times 10^{6}} \times \frac{22}{8} . F$-values thus represent estimates per-generation natural variation in gene expression calibrated by neutral mutational variation. The genome-wide CDC median $F$-value is $1.56 \times$ $10^{-4}$ (cf. [31]), indicating that variation among natural strains is roughly $10^{4}$-fold smaller than expected under mutation-drift equilibrium. (The median scaled natural and mutational variances are $2.40 \times 10^{-8}$ and $1.54 \times 10^{-}$ ${ }^{4}$, respectively.) With a maximum $F$-value of 0.23 , not a single gene shows evidence of positive selection for adaptive divergence at any timepoint. When tests are carried out for each gene at each timepoint (Figure 1A), $95.6 \%$ of hypotheses indicate stabilizing selection on expression level on average (FWER $<10^{-5}$ ). The nine natural $S$. cerevisiae lines in our study are estimated to have diverged between 3.02 and 4.19 thousand years ago ( $95 \%$ confidence interval); therefore $94.4 \%$ to $96.4 \%$ of 


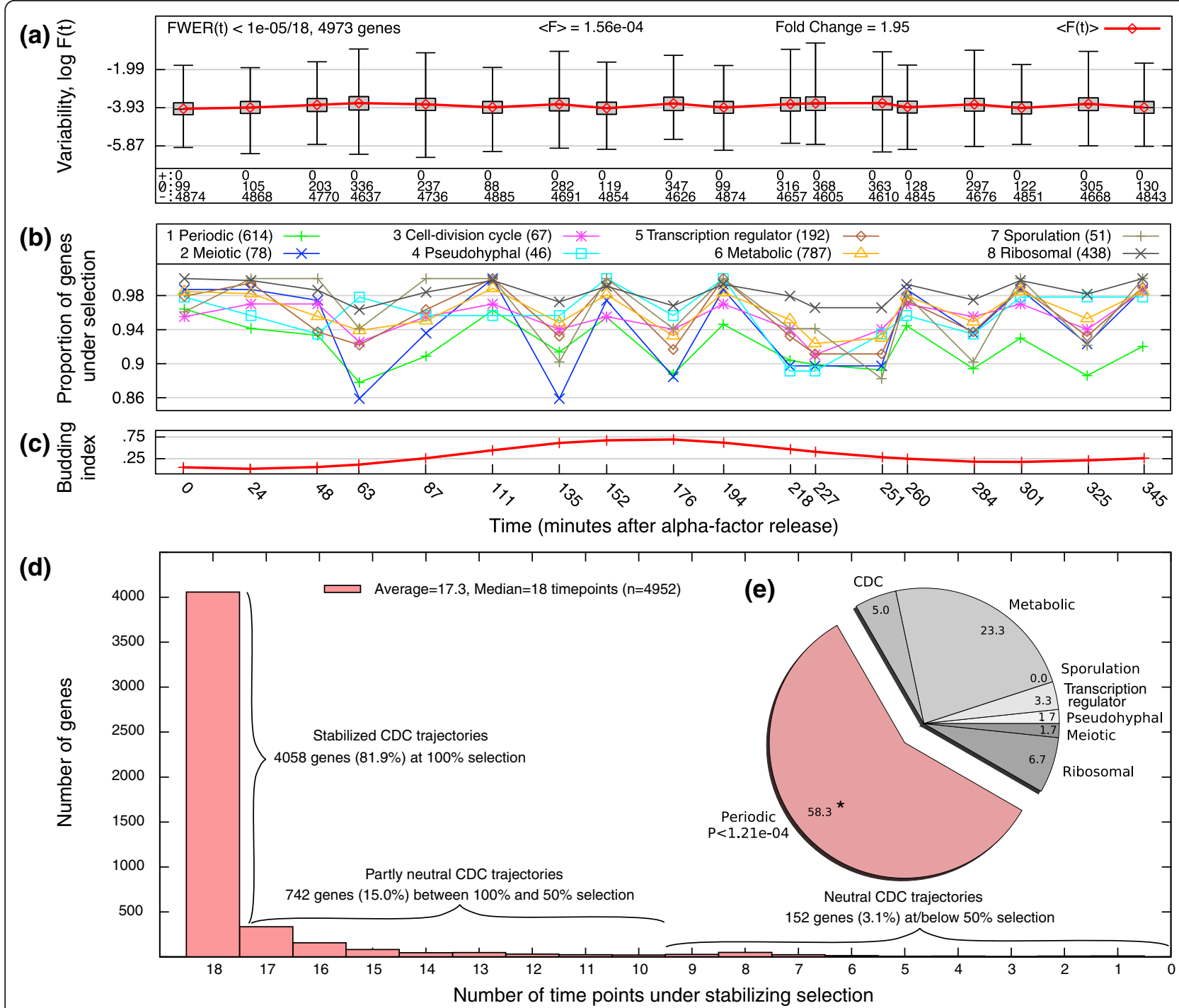

Figure 1 Natural variability in genome-wide gene expression. (a) Distributions of genome-wide gene expression variability $F(t)$ among natural S. cerevisiae strains across the cell-division cycle (CDC), and the number of genes exhibiting positive (+), stabilizing (-), or no selection (0) at each timepoint (FWER < 0.05). Average variability profile (red line) exhibits a maximum fold change of 1.95. (b) Proportion of genes under stabilizing selection over time for eight life-cycle terms, ranked by average proportion. Numbers of associated genes are shown in parentheses. See Figure S4 in Additional file 1 for profiles of GO Slim terms. (c) Average budding index for natural S. cerevisiae strains. (d) Histogram of the number of timepoints for which a gene's CDC-expression trajectory undergoes stabilizing selection, partitioned into stabilized, partly neutral, and neutral categories. (e) Enrichment of life-cycle terms among neutral genes. ${ }^{*}$ indicates significant enrichment $(F D R<0.05)$.

gene expression levels are under stabilizing selection. Moreover, the majority of genes (81.9\%) exhibit expression trajectories consistent with complete stabilizing selection at every timepoint, while 742 genes (15.0\%) exhibit low variability in at least half of the timepoints (partly neutral genes) and only 152 genes (3.1\%) exhibit neutral variability in at least half of the timepoints (neutral genes) (Figure 1D, Table S2 in Additional file 1). No single trajectory appears to diverge completely neutrally. Thus, when analyzed in terms of gene expression levels only without considering the effect of CDC-dynamics, the overall pattern of our data is consistent with previous hypotheses that the expression levels of most genes are under strong stabilizing selection.

One might suspect that the broad lack of expression divergence among strains may be due to a general deficiency of CDC-temporal variation for many of the genes. To test this, we partitioned S. cerevisiae expression variation into relative contributions from strain and temporal effects using a linear mixed model analysis. 3,750 genes $(59.9 \%)$ exhibit significant effects $(\mathrm{FDR}<0.1$ over all 6,251 $\times 2$ hypotheses): 2,797 genes $(46.6 \%)$ show 
significant strain variation (that is, divergence), 2,596 genes $(43.3 \%)$ show significant temporal variation, and 1,643 genes (26.2\%) show both effects. Averaging over these 1,643 genes, strain effects explain 39\% and temporal effects explain $23 \%$ of the total variance in gene expression; combining these marginal effects explains $50 \%-90 \%$ of each gene's total variance. Strain and temporal variances show significant but mild correlation $\left(R=0.25, P<10^{-10}\right.$; Figure S2 in Additional file 1), and temporal effects contribute $10^{4}$-fold more to overall expression variation compared to strain effects when scaled by divergence time (genome-wide medians $\sigma_{\text {time }}^{2}=9.54 \times 10^{-4}$ vs. $\left.\sigma_{\text {strain }}^{2}=7.43 \times 10^{-8}\right)$. Thus, considerable temporal variation in CDC-expression is present in the yeast transcriptome (see also Figure S3 in Additional file 1).

To relate evolutionary forces to yeast gene function, we computed the proportion of genes under stabilizing selection for eight broad life-cycle terms and $88 \mathrm{GO}$ Slim terms over time, $Q_{j}(t)$, where $j$ indexes each term. The $Q_{j}$ profiles of most terms appear qualitatively similar (Figure S4 in Additional file 1), and a comparison of average $Q_{j}$ values for life-cycle terms reveals that periodic, meiotic, and CDC-specific genes (in that order) are the most neutral (Figure 1B). In particular, a significant number of neutral genes are periodically expressed (Fisher's Exact test, FDR < 0.05; Figure 1E). Of the 88 GO Slim terms, only 5 terms have average $Q_{j}$ values less than 0.94 (the 95th percentile over $Q_{j}$; Table S3 in Additional file 1$)$ : helicase activity (0.76), extracellular region (0.86), cell wall (0.91), cellular component (0.92), and pseudohyphal growth (0.93). Of these, cell wall and extracellular region terms are enriched among the 1,643 genes with significant strain and time effects (FDR < 0.05). Thus, while it is not clear whether there is a functional aspect to expression divergence in temporal trajectories, among genes with the most strain divergence, specific functional categories are enriched within the set of temporally variable genes.

A hierarchical clustering of the entire CDC-transcriptome data set shows a complex inter-relationship among strains and timepoints, such that no strain's entire CDC-temporal expression and no timepoint's entire strain expression form a single clade (Figure S5 in Additional file 1); however, different timepoints from the same strain tend to be more similar than the same timepoints from different strains, indicating a general pattern of strain divergence. Notably, 17 of 18 timepoints for our S. paradoxus strain (YPS3395) cluster as a single clade, indicating their general distinction from S. cerevisiae expression. Yet only 457 genes (7.5\% of the genome) show significant differential expression between S. paradoxus and the 8 woodland S. cerevisiae lines $(t$-test, FWER $<0.1)$, and no gene shows greater than a three-fold change in expression level. Surprisingly, the $S$. cerevisiae laboratory strain exhibits the most divergent dynamic expression profile in this clustering, beyond the S. paradoxus outgroup, despite having only 248 genes (4\%) that are differentially expressed compared to woodland strains (FWER $<0.1$ ) with a maximum fold change of 4.2. Thus, compared to $S$. paradoxus, the laboratory $S$. cerevisiae strain shows only slightly greater expression level divergence from woodland strains but for fewer genes, yet it shows a more distinct pattern of temporal divergence. One possibility is that the laboratory strain's CDC molecular physiology has become adapted to laboratory growth conditions [32], which is manifest in its CDC-transcriptome dynamics. Overall, these results indicate that while levels of expression show limited among-strain and between-species divergence, the dynamic pattern of expression displays significant temporal fluctuations, with broad among-strain and between-species divergence.

\section{Divergence in CDC-temporal coexpression patterns is found at all scales of transcriptome organization}

To evaluate the quantitative divergence in CDC-temporal expression following the qualitative patterns revealed by clustering analysis above, we first generated a $6,082 \times 6,082$ gene coexpression matrix for each strain by computing pairwise correlations between all CDCtemporal gene expression profiles and then calculated matrix correlation coefficients between coexpression matrices for all pairs of strains (Figure S6A in Additional file 1). Due to the extreme size of the matrices, all comparisons yield significant concordance in coexpression patterns (FDR $<0.01)$, but the degree of concordance is low (avg. $R=0.11$ ), indicating most strains lack strong similarity in CDC-coexpression (that is, similar pairwise relationships between genes). Restricting these coexpression matrices to a subset of 266 transcriptional regulatory genes does not strengthen this pattern of weak association (avg. $R=0.12$; Figure S6B in Additional file 1). Controls using replicated and simulated microarray data confirm this pattern (Text S1). As may be expected, $S$. paradoxus has the lowest coexpression correlation with other strains (avg. $R=0.047$ ); however, S. cerevisiae strains YPS3137 and YPS2073 also have low correlations (0.055 and 0.068). The laboratory strain shows an average correlation of 0.12 , indicating that its divergence in CDC-coexpression is typical compared to woodland strains. Thus, the laboratory strain appears to show pronounced divergence in overall CDC-transcriptome dynamics compared to other strains (see above) without markedly different coexpression relationships (that is, changes in regulation). Overall, we found 
considerable divergence in the genome-wide pattern of temporal coexpression.

To assess coexpression divergence in a time-specific manner, we grouped each strain's expression data into three overlapping CDC-phase groups (first, middle, and last nine timepoints). We first assessed coexpression matrix similarity between strains and between CDCphase groups. This recapitulated the pattern of weak association between strains $(R=0.075$; Figure $2 \mathrm{~A})$. Coexpression matrices consistently cluster by strain (Figure 2B), but cluster relationships between strains are unique to each $\mathrm{CDC}$-phase group (Figure $2 \mathrm{C}$ ). We also identified phase-directions of temporal covariation using a singular value decomposition (SVD) of each strain's expression data for each of the three CDC-phase groups.
Within each group, the angular distance of major phasedirections between strains averages $75.8^{\circ}$, close to the maximum of $90^{\circ}$ (Figure S7A in Additional file 1). Multidimensional scaling (Figure S7C in Additional file 1) and hierarchical clustering (Figure S7D in Additional file 1) indicate that similarity relationships between strains are phase-specific. These results indicate that the genomewide pattern of coexpression divergence is time-dependent.

Since coexpression divergence may occur at different scales of transcriptome organization, we also assessed the pattern of modular temporal coexpression. We defined a coexpression $k$-module for every gene as its $k$ most correlated genes within each strain. We assessed divergence in modular coexpression by computing the overlap of each gene's $k$-modules between strains and

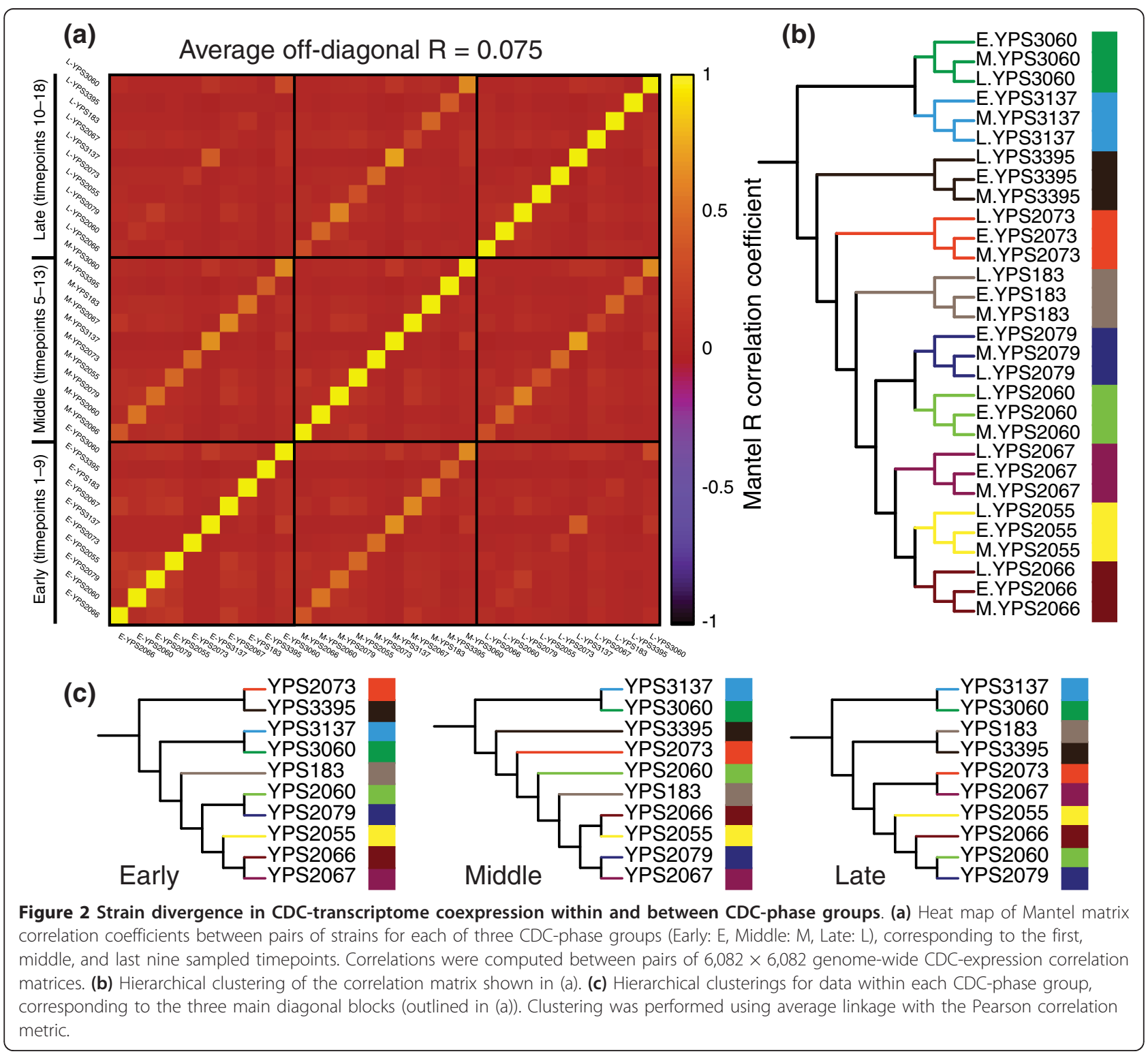


determining the degree of excess overlap compared to random expectation among significant genes. Less than two-thirds of genes exhibit significant overlap at any scale (from $25 \%$ at $k=25$ to $65 \%$ at $k=2,500$, averaging over all strain pairs, $P<1 / 250$ ), suggesting that patterns of shared temporal coexpression cannot be identified for a large portion of the genome. While the average overlap among significant genes is consistently greater than expected by chance (Figure S8 in Additional file 1), the excess is generally low, averaging $8.24 \%$ with a minimum of $4.39 \%$ at $k=25$ and maximum of $10.03 \%$ at $k=$ 880 genes (Table 1). Thus, similar to the matrix correlation results, the pattern of modular coexpression shows low concordance between strains regardless of scale. Moreover, there is lower overlap at smaller scales, suggesting that temporal coexpression diverges more rapidly for genes that are more tightly coexpressed within a genome. To determine whether relationships of modular coexpression between strains change across organizational scales, we computed hierarchical clusterings of the $10 \times 10$ matrices of average module overlap between strains (Figure S9 in Additional file 1). A few strains, notably YPS3137 and YPS2073, show changes in overlap relationships across scales, suggesting that these strains differ in temporal coexpression at all scales of transcriptome organization. Thus, divergence in CDCtemporal coexpression is found genome-wide, in a timedependent manner, and at all scales of transcriptome organization.

\section{CDC regulatory architecture exhibits time-dependent changes in multi-dimensional complexity}

The gene-oriented analyses above indicate surprisingly large divergence in CDC-temporal expression,

Table 1 Strain divergence in modular coexpression structure

\begin{tabular}{cccc}
\hline $\begin{array}{c}\text { Diameter } \\
\text { (\%) }\end{array}$ & $\begin{array}{c}\text { Sig. modules } \\
\text { (\%) }\end{array}$ & $\begin{array}{c}\text { Overlap (\% of } \\
\text { diameter) }\end{array}$ & $\begin{array}{c}\text { Excess } \\
\%\end{array}$ \\
\hline $25(0.4)$ & $1507.3(24.8)$ & $1.2(4.8)$ & 4.39 \\
$100(1.6)$ & $1645.7(27.0)$ & $10.6(10.6)$ & 8.96 \\
$500(8.2)$ & $3220.4(52.9)$ & $88.0(17.6)$ & 9.38 \\
$880(14.5)$ & $3389.2(55.7)$ & $215.6(24.5)$ & 10.03 \\
$1314(21.6)$ & $3625.3(59.6)$ & $408.6(31.1)$ & 9.49 \\
$2500(41.1)$ & $3972.1(65.3)$ & $1207.5(48.3)$ & 7.20 \\
\hline
\end{tabular}

A module is defined for every gene as the set of its $k$ top correlating genes by Pearson correlation of temporal expression profiles, where $k$ is the diameter, shown as number of genes and as genome-wide proportion (of 6,082 genes). Sig. modules reports the number and percentage of significant gene modules $(P<1 / 250)$ averaged over all pairs of strains. Overlap reports the number of genes overlapping for a given module between a pair of strains, at the specified diameter $k$, averaged over all significant modules and all pairs of strains. This is also shown in parentheses as a percentage of diameter. Excess shows the excess percentage of overlap compared to random expectation using binomial sampling. The excess percentage averaged over all $k$ is $8.24 \%$. suggesting a broad potential for evolutionary divergence of expression dynamics despite stabilizing selection on expression levels. Changes in expression dynamics imply changes in the timing patterns of genome-wide gene regulation. To dissect the architecture of time-dependent gene regulation that underlies the observed pattern of transcriptome divergence, we analyzed multivariate (multi-genic) patterns of expression covariation among the $S$. cerevisiae lines, including time-dependent multivariate patterns. We first performed a canonical correlation analysis using genome-wide expression grouped by timepoint and found that expression can be correlated nearly perfectly between all pairs of timepoints using primary canonical variables $(R \approx 1.0$, FWER $<0.05)$. This indicates that genome-wide expression at each timepoint shares the same sub-space (that is, fundamental directions of variation); however, particular directions of major variation may differ across timepoints. We next assessed the degrees of freedom of expression variation among strains by analyzing the covariation at each timepoint independently, using latent factor mixed model analysis (LFA) and principal component analysis (PCA). Compared to patterns seen in the mutation accumulation lines, natural time-specific covariation exhibits greater overall regulatory complexity, averaging 4.6 vs. 2 factors by LFA (Table S4 in Additional file 1), and restricted degrees of freedom of covariation, averaging 6.1 vs. 13 dimensions by PCA (Figure S13A in Additional file 1), at each timepoint. Combining all timepoints and strains, a total of 56 dimensions are required to explain $90 \%$ of the covariation in the natural strain CDC data (Figure 3). Surprisingly, these degrees of freedom do not simply separate into time and strain components: if each strain's expression is time-averaged, only five PCA factors explain the resulting among-line covariation; if each timepoint's expression is strain-averaged, ten factors explain the among-timepoint covariation. Thus, a much greater complexity of expression divergence is revealed when both $\mathrm{CDC}$-temporal and strain covariation are taken into account.

Both LFA and PCA results strongly suggest the presence of differential constraints on transcriptome divergence as a function of CDC progression. We examined this by asking whether yeast strain covariance structure changes between different timepoints. We applied a SVD to the expression data at each timepoint for all $S$. cerevisiae strains, obtaining $r=9$ multivariate directions of strain divergence $U^{r}(t)$ for each of the 18 timepoints $t$ [33] (see Supplemental materials and methods). We call these CDC-directions, which might reflect developmental constraints, mutational biases, or directions of selection (or combinations thereof), for example. We first computed angular distance between the major 


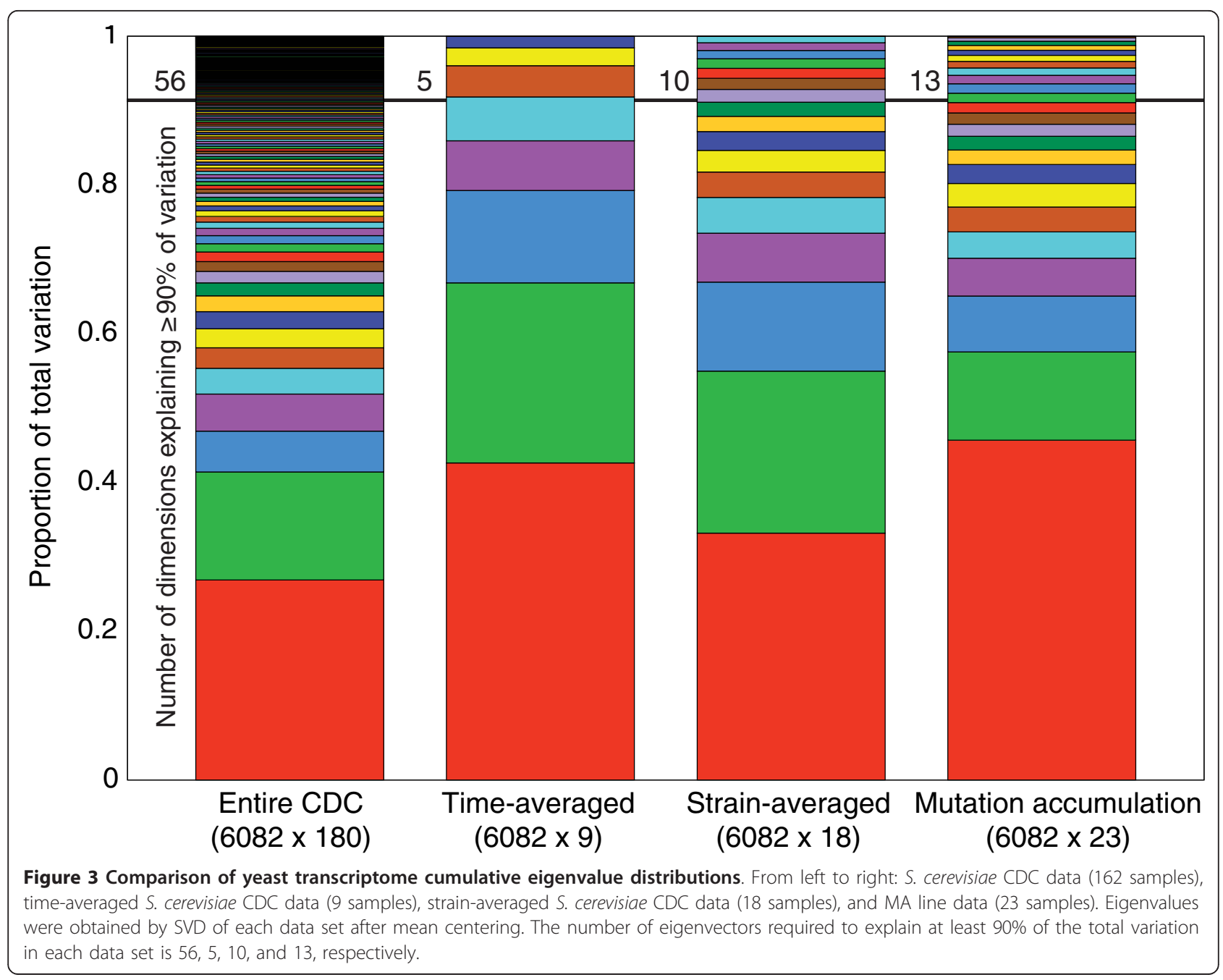

CDC-directions for all timepoint pairs $\left(\angle U^{1}(s) U^{1}(t)\right.$; Figure $4 \mathrm{C}$ ). Adjacent timepoints as well as those in phase between cell-division cycles appear more similar than other timepoints, indicating that changes in covariance structure are both gradual and cyclic. Despite these similarities, angles average $50.4^{\circ}$ and range from $19.4^{\circ}$ to $88.9^{\circ}$. A random angles test failed to identify any significantly small angles (that is, significantly similar directions), even with a lenient cutoff (FWER < 0.15). Visualization of the major CDC-direction distance matrix by multidimensional scaling reiterates this pattern (Figure 4A). These results suggest that most major CDC-directions are distinct. Similar testing of each of the eight minor CDC-directions (Figure 4D) identified only eight significantly small angles out of 1,072 comparisons. Common principal component analysis of time-dependent covariation [34] revealed broadly consistent results (Text S2). Thus, we observe significant changes in the yeast transcriptome covariance structure across strains throughout the CDC.
To assess whether the CDC-directions correspond to biologically relevant axes of covariation, we identified the genes contributing the most to strain covariation in each major CDC-direction by correlation and determined the functional terms enriched among the top 5\% of genes (Tables S6, S7 in Additional file 1). Significant terms vary by timepoint and include metabolic, periodic, ribosomal, and CDC life-cycle terms (FDR $<0.05)$. In addition, TATA regulatory motifs have been hypothesized to drive expression divergence via neutral drift [31]. We found that TATA-associated genes project onto major CDC-directions 4-fold less than genes lacking TATA motifs, which are over-represented among the top $5 \%$ of genes $(P<0.01$, Table S8 in Additional file 1). Also, few of the 152 genes with neutral CDCexpression are found among the top $5 \%\left(P<10^{-5}\right)$. This paucity of genes hypothesized to diverge neutrally argues against drift as a major force in strain diversification of CDC-directions. We also tested whether the major CDC-directions (of within-species covariation) are 


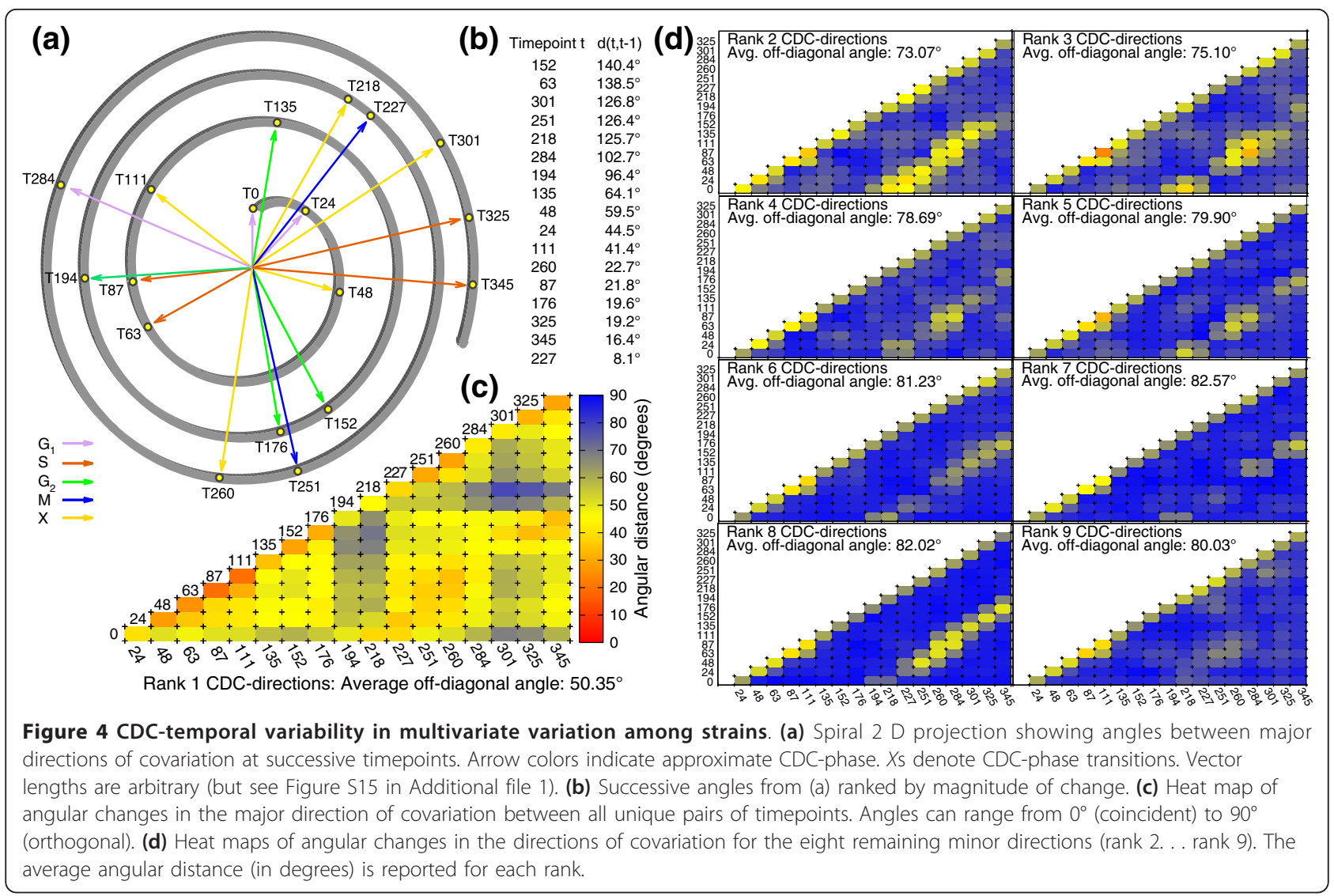

predictive of directions of between-species divergence, as might be expected for neutral species divergence [35]. For each timepoint we calculated angular distance between the major S. cerevisiae CDC-direction and the displacement vector of $S$. paradoxus expression, oriented within S. cerevisiae CDC-space (for example, Figure S14 in Additional file 1). All angles exceed $45^{\circ}$, and no angle is significantly small (FWER $<0.15$ ). Thus, withinspecies covariation does not predict the direction of between species divergence. However, release from $\alpha$-factor, S-phase, and the $G_{2} / M$ transition have the smallest angles, suggesting that response to mating pheromone and DNA replication dynamics may be more constrained in evolutionary covariation.

We next evaluated whether the amount of variation projected onto the multivariate $\mathrm{CDC}$-directions reveals a different, non-stabilizing pattern of selection compared to the pattern for individual genes. We computed $F$-statistics by comparing natural and mutational among-line expression variances projected onto each timepoint's CDC-directions. Although the average $F$-value over major CDC-directions $U^{1}(t)$ is 14.6-fold larger than the genome-wide average $F$-value $\left(2.28 \times 10^{-3}\right.$ vs. $1.56 \times$ $\left.10^{-4}, P=1.5 \times 10^{-4}\right)$, all $F$-values remain significantly low, including those calculated for minor CDC- directions (FWER < 0.05). Therefore, multivariate patterns of transcriptome divergence are also consistent with stabilizing selection. However, the temporal profile of major multivariate $F$-values, unlike that for individual genes, exhibits peaks in expression variability $(87,176$, 260, and 345 min.; Figure S15 in Additional file 1); the average peak is 1.4 -fold greater than that at all other timepoints $(P=0.018)$ and 19.1 -fold greater than the genome-wide average $(P=0.006)$. Intriguingly, these peaks in expression variability are preceded by large changes in the major axis of CDC-covariation $(63,152$, 251, and $301 \mathrm{~min}$.), occur just prior to CDC-phase transitions $(97,218,267$, and approximately $350 \mathrm{~min}$.), and coincide with drops in regulatory complexity (latent factors; 176, 260, 345 min.) (Table S4 in Additional file 1; see also Figure 4B). In addition, reductions in regulatory complexity generally coincide with the CDC-phase transitions $G_{1} / S, G_{2} / M$, and $M / G_{1}(48,218,260,301 \mathrm{~min}$.; except $S / G_{2}$ at $111 \mathrm{~min}$.), suggesting greater constraint on gene regulation through the influence of CDC checkpoints. Thus, temporal fluctuations in strain variability might reflect multi-genic pleiotropic effects being channeled to varying dimensions and directions of gene expression through a regulatory architecture that changes dynamically across CDC-phases [7]. 
Heterochronic changes in expression timing explain strain divergence for the majority of yeast genes

Our multivariate analysis of the architecture of genomewide gene regulation argues that the broad pattern of CDC-transcriptome divergence among yeast strains is heavily influenced by dynamical changes in control. However, if this architecture of timing control involves a global cascade of regulation, any changes in control could cause broad negative pleiotropic effects throughout the CDC [8]. Given our findings of strong stabilizing selection on both univariate and multivariate strain variation across the CDC, such a global, hierarchical architecture seems unlikely. Alternatively, this architecture may be organized into discrete modules of regulation that exhibit dynamically-autonomous timing control [36]. Moreover, superposition of regulatory timing patterns from different modules could combinatorially generate the regulatory complexity required for transcriptome-wide timing control while minimizing negative pleiotropic effects.

We evaluated this hypothesis of modular timing control by identifying genes that share patterns of expression heterochrony (evolutionary shifts in expression timing compared to the CDC) $[27,37,38]$, which can be used to delineate dissociable units of structure and function [29,39]. Briefly, we reasoned that if two genes are coregulated, their temporal expression trajectories might show similar evolutionary shifts in timing between strains and species, despite overt differences in the expression trajectories themselves. We tested for the presence of heterochrony in the yeast cell-division cycle by asking whether a time transformation (that is, heterochrony) model significantly explains a gene's divergence in temporal expression between two strains (Figure 5A). On average, our heterochrony model explains 61\% of between-strain transcriptome variation (Figure 5B).

(a) Heterochrony model: $\mathrm{y}(\mathrm{t})=\mathrm{A}+\mathrm{Bx}((\mathrm{Beta}(\mathrm{t}, \alpha, \beta)+\gamma) \bmod 1)+\varepsilon$ Time-independent model: $y(t)=A+B x(t)+\varepsilon$

(b)

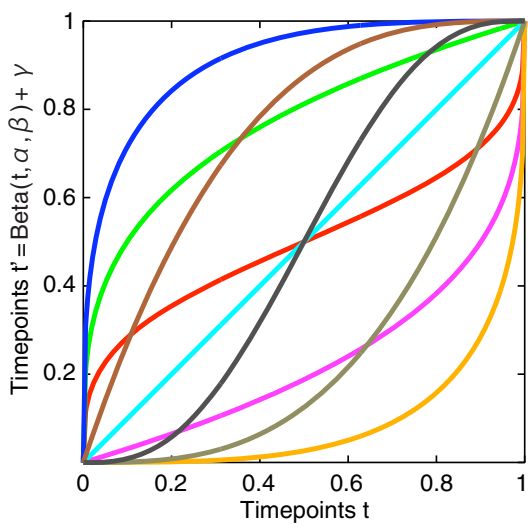

(c)

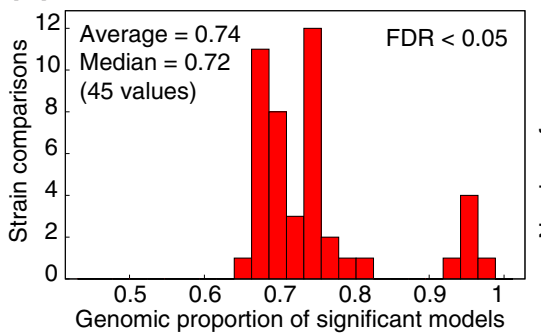

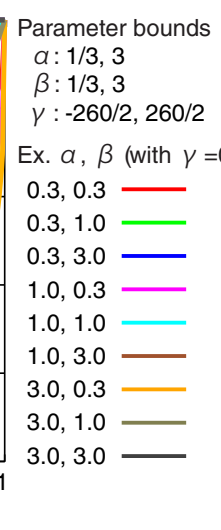

(d)

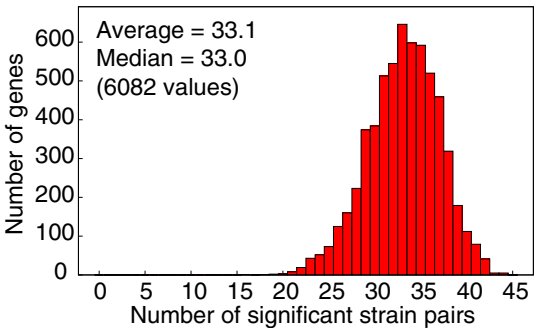

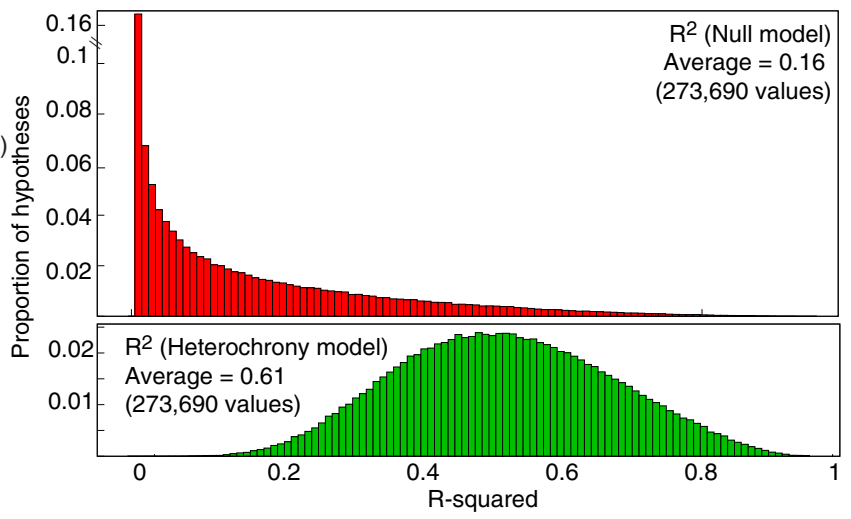

(e)

Figure 5 The heterochrony model of time-dependent changes in gene expression trajectories between strains. The model was fit to single period, Z-standardized CDC-expression data for a single gene measured in two strains. (a) Formulation of the time-independent (null) and heterochrony regression models. The heterochrony model estimates a timepoint mapping between strains using the Beta cumulative distribution function, which generates smooth and invertible transformations on $[0,1]$ according to parameters $\alpha$ and. $\beta$. This model also allows translation of expression trajectories using the phase parameter $\gamma$. Transformed timepoints were modulated around 1 , so that transformations are defined with respect to a single cell-division cycle. Estimates of $\alpha, \beta$, and $\gamma$ were bounded within [1/3, 3], [1/3, 3], and [-260/2, 260/2], respectively, where 260 is the CDC period. The light blue line $(\alpha=1 ; \beta=1 ; \gamma=0)$ describes the null (time-independent) model, where $t=t^{\prime}=$ Beta $(t, 1,1)+0$. (b) Distributions of $R^{2}$ values for the time-independent (top) and heterochrony (bottom) models, over all 45 comparisons per gene. Both models were fit identically, except that parameter values for the null model were fixed at $(\alpha=1 ; \beta=1 ; \gamma=0)$. (c) Distribution of the proportion of significant $F$-values (genes) over the 45 strain comparisons (FDR $<0.05$ ). (d) Distribution of the number of significant strain comparisons over genes. (e) The number of genes significant in at least $k$ comparisons versus $k$. A cutoff of $30 / 45=2 / 3$ was used to classify a subset of 4998 genes as heterochronic. 
We then computed a likelihood-ratio statistic for every gene by comparing the fit of the heterochrony model to the fit of a time-independent model. $64 \%-96 \%$ of genes show a significant time effect for any between-strain comparison (d.f.1, 3 and 14, FDR < 0.05; Figure 5C), indicating a broad pattern of heterochronic divergence. Each gene exhibits significant fit to the heterochrony model for an average of 33.1 of the $\left(\begin{array}{c}10 \\ 2\end{array}\right)=45$ pairwise comparisons (Figure 5D). We retained 4998 genes showing consistent support for heterochrony ( $\geq 2 / 3$ significant comparisons; Figure $5 \mathrm{E}$ ) for the analysis of shared patterns of heterochrony. As expected, these genes tend to exhibit large dynamical fluctuations in expression level across the CDC: $85.8 \%$ belong to the set of 2,596 genes with significant temporal variation $\left(P<10^{-10}\right)$. At least $85 \%$ of the top 1,000 periodically expressed genes in our data set show significant heterochrony (Figure S16 in Additional file 1). In addition, functional analysis reveals significant enrichment for a variety of GO Slim terms (Text S3). These results suggest that the major mode of transcriptome divergence in the yeast CDC entails changes in timing (heterochrony) rather than changes in levels (heterometry) of expression.

\section{Shared patterns of heterochrony reveal modular timing changes}

We identified shared patterns of heterochrony among the 4,998 heterochronic genes by comparing their timing change curves (defined by the heterochrony model parameter estimates; Figure S17 in Additional file 1), such that two genes are similar if their timing change curves are concordant across the entire CDC (Figure S19 in Additional file 1). In this way we computed a distance matrix that characterizes the timing pattern relationships between all pairs of genes (Text S4). Clustering genes by their timing pattern relationships revealed seven significant timing modules, consistent with the hypothesis of modular timing control (Text S5). To identify the genes significantly associated with each timing module, we performed a pairwise analysis by counting the number of between-strain comparisons (out of 45) in which two genes exhibit the same pattern of timing change. We identified 5,393 significant interactions connecting 3,715 genes (binomial, $P<10^{-4}$; see Additional file 2); $47.2 \%$ of the significant interactions connect genes within the same timing module. Genes sharing significant interactions display an average similarity of 0.46 , compared to the genome-wide average similarity of 0.19 (Figure S24 in Additional file 1). Interacting genes also share functional ontology terms, on average sharing $95 \%$ of possible life-cycle terms $(P<$ $\left.10^{-7}\right)$ and $23 \%$ of possible GO Slim terms $\left(P<10^{-19}\right)$, consistent with a functional interpretation for divergence in expression timing. We partitioned genes sharing significant heterochronic interactions into two groups: 1,828 genes showing a majority of interactions within an individual timing module (module-specific genes), and 1,887 genes showing a majority of interactions across timing modules (between-module genes). Among these 3,715 genes, within-module interactions are found 5.6-fold more often than between-module interactions $\left(P<10^{-10}\right)$, indicating that module-specific genes comprise the inter-connected core of each timing module (Figure 6A). Functional enrichment of timing modules reveals five life-cycle terms and 21 GO Slim terms associated with four of the seven timing modules (Table S10 in Additional file 1), whereas analysis of between-module genes revealed no significantly enriched terms (FDR < 0.1). Thus, analysis of shared patterns of heterochrony reveals significant modular organization in the timing patterns of genome-wide gene expression and suggestive evidence that these modules are associated with cellular function.

\section{Modular timing changes reflect coherent and dynamically-autonomous timing control}

Heterochronic modularity of gene expression timing suggests that each timing module could represent a distinct unit of temporal development, responsible for executing a particular timeline of gene expression events. In this case, each module's characteristic timing pattern might undergo dynamically-autonomous evolution without losing coherence in modular timing control. According to this hypothesis, a module's timing pattern may change during evolutionary divergence, increasing variation among modules; however, variation in the timing patterns of genes within a module should not change (or change more slowly), since this implies potentially deleterious changes in functional coregulatory relationships. We first used analysis of variance to test for differences in the mean timing pattern among modules, using the timing change curves of module-specific genes pooled from the 45 strain comparisons. Timing patterns differ significantly among modules $\left(P<10^{-10}\right)$, suggesting that timing modules undergo heterochronic divergence in a dynamically-autonomous manner. We then examined timing pattern variability within modules, by comparing the observed variance in timing change curves among module-specific genes to a distribution of random variances, produced by grouping timing change curves drawn randomly from the set of all observed curves. Within-module timing pattern variability is generally lower than expected and may be lower within species than between species (Text S6 and Figure S26 in Additional file 1). Linear discriminant analysis of the timing pattern relationships for module-specific genes illustrates 


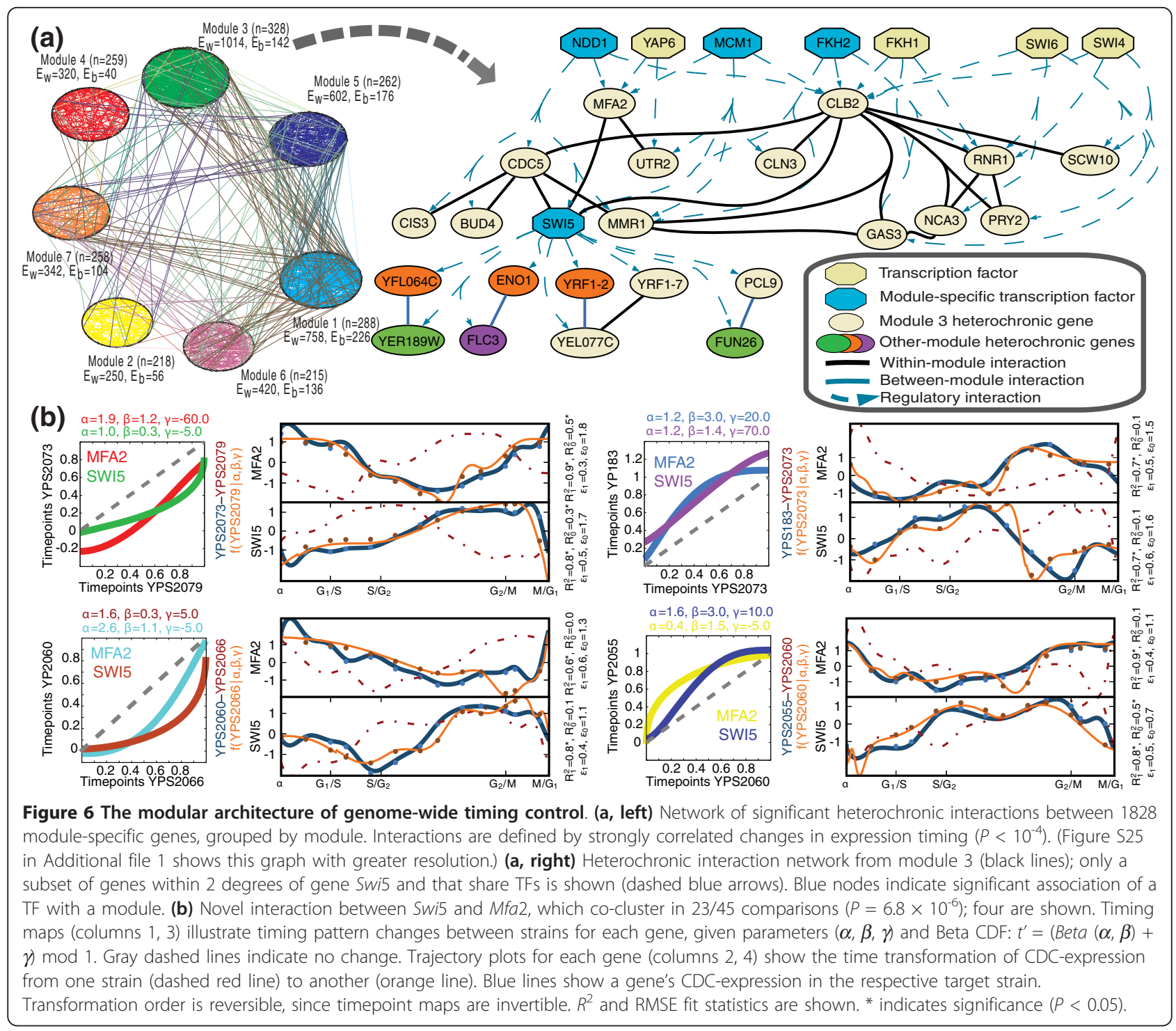

this coherence of timing patterns within modules despite differences between modules (Figure 7). These results suggest that divergence in timing patterns may increase more quickly between modules than within modules, consistent with the representation of modules as distinct units of timing control.

Furthermore, robustness of the yeast CDC against genetic [40], environmental [41], and dynamical perturbations [42] suggests the possibility that timing pattern variability both within and between modules might be limited by a form of negative selection, potentially canalizing selection [43-45], which could reinforce the coherence of modules as integrated developmental processes. Consistent with this, module-specific genes as a group show significantly low variation for timing change curves across strain comparisons $(P=0.0002)$, and when separated by module, their strain variation correlates with each module's estimated coherence (Spearman's $r=-0.94, P=0.0009)$. This suggests a relationship between within-module variability and among-strain variability in timing patterns (Text S7). In addition, variability among all timing patterns is also lower than expected and is time-dependent, suggesting the possibility of system-wide coordination and periodic synchronization of modular timing patterns (Text S8 and Figure S27 in Additional file 1). These results suggest that the CDC timing control architecture is comprised of a core of distinct, coherent, and dynamicallyautonomous modules involving nearly $30 \%$ of the genome, combined with a layer of interactions between modules, which may potentially coordinate or synchronize expression timing globally. 


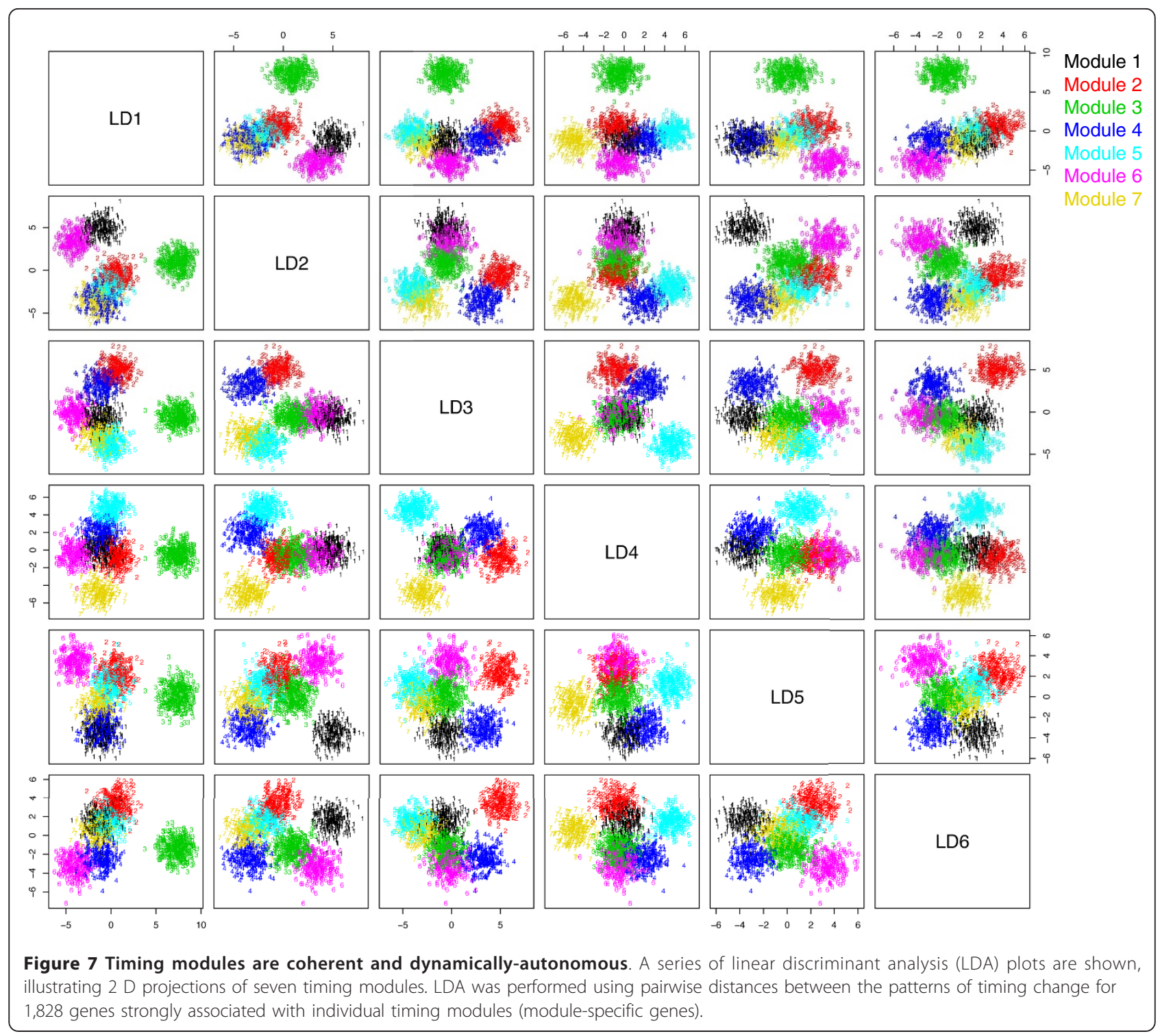

\section{Heterochronic expression of module-specific regulatory factors may explain modular timing changes}

While the prevalence of heterochrony is consistent with broad changes in gene coregulation, modularity in the patterns of heterochrony suggests that regulatory architecture itself could effectively constrain multi-genic strain variation into distinct channels of phenotypic expression. In this way, widespread divergence in transcriptome dynamics may be explained by predominantly quantitative changes in the expression patterns of module-specific regulatory factors, rather than qualitative changes in gene coregulation. Using the 1828 modulespecific genes, we tested whether strongly shared heterochrony implies common transcription factor trans-regulation, as one possible mode of module-specific gene regulation. Genes sharing heterochronic interactions share more TFs than expected $\left(P<10^{-100}\right)$ and associate with TFs more strongly than pairs of genes without strongly shared heterochrony $\left(P<10^{-10}\right)$. The genomewide pattern of TF-gene trans-regulatory interactions also associates significantly with the segregation of genes into timing modules $(P=0.014)$. We then sought to identify TFs that associate specifically with each timing module, using $2 \times 2$ contingency tables to summarize the interactions between each TF and module (Text S9). We identified 37 TFs showing 42 module-specific associations, averaging six TFs per module (FDR $<0.1$ ); this represents significant association for $59 \%$ of the 63 TFs tested (the subset of 117 TFs showing $\geq 7$ targets [46]). These 37 module-specific TFs themselves exhibit significant patterns of heterochrony (Table 2; Figure S28 in Additional file 1); as a class, they show more extreme 
Table 2 Heterochrony in module-specific transcription factors

\begin{tabular}{|c|c|c|c|c|c|c|}
\hline & Gene (Alias) & $P$ & $\overline{R_{H_{1}}^{2}}$ & $\overline{R_{H_{0}}^{2}}$ & Sig. F-tests (Prop.) & Distortion \\
\hline \multirow[t]{7}{*}{ Module 1} & YHR206W (Skn7) & $0.06567^{\circ}$ & 0.591 & 0.138 & $36(0.80)$ & 77.48 \\
\hline & YPL089C (RIm1) & $0.07051^{\circ}$ & 0.605 & 0.142 & $35(0.78)$ & 75.80 \\
\hline & YNL309W (Stb1) & $0.06356^{\circ}$ & 0.616 & 0.146 & $36(0.80)$ & 74.13 \\
\hline & YNL216W (Rap1) & $0.02399^{*}$ & 0.624 & 0.175 & $33(0.73)$ & 73.92 \\
\hline & YLR403W (Sfp 1) & $0.01056^{*}$ & 0.653 & 0.200 & $36(0.80)$ & 73.46 \\
\hline & YDR207C (Ume6) & $0.01626^{*}$ & 0.532 & 0.153 & $27(0.60)$ & 63.20 \\
\hline & YPR104C (Fh/1) & $0.0015^{* *}$ & 0.547 & 0.120 & $28(0.62)$ & 59.81 \\
\hline \multirow[t]{2}{*}{ Module 2} & YKL112W (Abfi) & $0.025^{*}$ & 0.629 & 0.201 & $33(0.73)$ & 68.51 \\
\hline & YIL131C (Fkh1) & $0.04142^{*}$ & 0.649 & 0.151 & $35(0.78)$ & 62.44 \\
\hline \multirow[t]{8}{*}{ Module 3} & YOL028C (Yap7) & $0.09046^{\circ}$ & 0.659 & 0.163 & $36(0.80)$ & 77.63 \\
\hline & YDR123C (Ino2) & $0.03916^{*}$ & 0.585 & 0.134 & $33(0.73)$ & 76.02 \\
\hline & YOR372C (Ndd1) & $0.01219^{*}$ & 0.592 & 0.120 & $34(0.76)$ & 73.02 \\
\hline & YNL068C (Fkh2) & $0.00457^{* *}$ & 0.601 & 0.119 & $34(0.76)$ & 71.61 \\
\hline & YER040W (Gln3) & $0.00459^{* *}$ & 0.593 & 0.161 & $29(0.64)$ & 70.99 \\
\hline & YMR043W (Mcm1) & $0.01094^{*}$ & 0.672 & 0.203 & $40(0.89)$ & 69.36 \\
\hline & YGL237C (Hap2) & $0.00053^{* * *}$ & 0.537 & 0.138 & $22(0.49)$ & 69.01 \\
\hline & YML007W (Yap1) & $0.03816^{*}$ & 0.603 & 0.136 & $35(0.78)$ & 62.86 \\
\hline \multirow[t]{5}{*}{ Module 4} & YOR028C (Cin5) & $0.03329^{*}$ & 0.582 & 0.165 & $29(0.64)$ & 87.10 \\
\hline & YPL202C (Aft2) & $0.07004^{\circ}$ & 0.579 & 0.092 & $34(0.76)$ & 75.80 \\
\hline & YDL056W (Mbp1) & $0.05184^{\circ}$ & 0.588 & 0.158 & $33(0.73)$ & 73.87 \\
\hline & YGL071W (Rcs1) & $0.02813^{*}$ & 0.581 & 0.115 & $33(0.73)$ & 73.42 \\
\hline & YDL106C (Pho2) & $0.01764^{*}$ & 0.569 & 0.143 & $27(0.60)$ & 61.63 \\
\hline \multirow[t]{7}{*}{ Module 5} & YPR065W (Rox1) & $0.06635^{\circ}$ & 0.666 & 0.185 & $35(0.78)$ & 80.97 \\
\hline & YBR049C (Reb1) & $0.04439^{*}$ & 0.682 & 0.206 & $37(0.82)$ & 80.96 \\
\hline & YDR423C (Cad1) & $0.05867^{\circ}$ & 0.574 & 0.119 & $31(0.69)$ & 75.47 \\
\hline & YHR178W (Stb5) & $0.09263^{\circ}$ & 0.626 & 0.166 & $37(0.82)$ & 70.27 \\
\hline & YMR037C (Msn2) & $6.0 \times 10^{-5 * * *}$ & 0.597 & 0.183 & $35(0.78)$ & 72.13 \\
\hline & YMR070W (Mot3) & $0.09263^{\circ}$ & 0.624 & 0.161 & $35(0.78)$ & 60.97 \\
\hline & YKL062W (Msn4) & $0.02334^{*}$ & 0.706 & 0.242 & $36(0.80)$ & 54.91 \\
\hline \multirow[t]{7}{*}{ Module 6} & YNL216W (Rap1) & $0.05212^{\circ}$ & 0.624 & 0.175 & $33(0.73)$ & 73.92 \\
\hline & YJR060W (Cbfi) & $0.00544^{* *}$ & 0.605 & 0.140 & $35(0.78)$ & 73.15 \\
\hline & YIR018W (Yap5) & $0.00379^{* *}$ & 0.527 & 0.110 & $27(0.60)$ & 70.80 \\
\hline & YDL020C (Rpn4) & $0.08764^{\circ}$ & 0.641 & 0.185 & $30(0.67)$ & 70.10 \\
\hline & YOR344C (Tye7) & $0.03477^{* *}$ & 0.663 & 0.175 & $38(0.84)$ & 68.96 \\
\hline & YKL112W (Abfi) & $0.04084^{*}$ & 0.629 & 0.201 & $33(0.73)$ & 68.51 \\
\hline & YPR104C (Fh/1) & $0.02139^{*}$ & 0.547 & 0.120 & $28(0.62)$ & 59.81 \\
\hline \multirow[t]{6}{*}{ Module 7} & YBR049C (Reb1) & $0.00056^{* * *}$ & 0.682 & 0.206 & $37(0.82)$ & 80.96 \\
\hline & YKR099W (Bas1) & $0.01578^{*}$ & 0.591 & 0.163 & $29(0.64)$ & 69.26 \\
\hline & YBL103C (Rtg3) & $0.04638^{*}$ & 0.607 & 0.144 & $36(0.80)$ & 67.13 \\
\hline & YDR043C (Nrg1) & $0.08764^{\circ}$ & 0.637 & 0.205 & $33(0.73)$ & 63.85 \\
\hline & YDR146C (Swi5) & $0.02932^{*}$ & 0.764 & 0.289 & $41(0.91)$ & 63.56 \\
\hline & YDL106C (Pho2) & $0.02567^{*}$ & 0.569 & 0.143 & $27(0.60)$ & 61.63 \\
\hline
\end{tabular}

$2 \times 2$ TF-module association tables were computed that reflect the number of module-specific genes $(n=1,828)$ that associate with one of seven timing modules and are regulated by one of 63 transcription factors (TFs). (A subset of 63/117 TFs were used that has at least seven targeted genes.) TF regulatory binding data were obtained from [46] using a cutoff of $\mathrm{P}<0.001$ and moderate conservation (cons $=1$ ). Fisher's Exact tests were used to evaluate the significance of each TF-module association along with a false discovery rate correction ***indicates FDR $<0.001$; **indicates FDR $<0.01$; ${ }^{*}$ indicates FDR $<0.05$; ${ }^{o}$ indicates FDR $<0.1$. In total 37 TFs show significant modular association (FDR < 0.1). Five TFs associate with two modules $\left(A b f 1\right.$, Fhl1, Pho2, Rap1, Reb1). $R_{H}^{2}$ and $\overline{R_{H}^{2}}$ indicate explained CDC-expression variation averaged over 45 strain comparisons, computed by the time-dependent heterochrony or timeindependent model, respectively. Sig. F-tests indicates the number (and proportion) of significant $F$-tests supporting the heterochrony model, among strain comparisons. Distortion is computed as the RMSE of the optimal time transformation curve against a line $(\alpha=1, \beta=1, \gamma=0)$, averaged over strain comparisons. Genes are ranked by distortion for each category. The average genome-wide distortion $(n=6.082)$ is 67.6 with a standard deviation of 8.7 . 
heterochronic shifts (distortion) compared to expectation from all heterochronic genes (76th percentile) and from all TFs (76th percentile). At least one TF from every module shows significantly large distortion compared to all heterochronic genes or all regulatory factors $(P<0.05)$; however, only one of these TFs $(\operatorname{Cin} 5)$ is among the top 50 of all heterochronic genes genome-wide (rank-46 by distortion; Table S9 in Additional file 1). There do not appear to be differences in the distortion of these TFs among modules (ANOVA, $P=0.2$ ). Thus, quantitative, heterochronic changes in the expression patterns of module-specific regulatory factors may drive divergence in CDC-transcriptome dynamics. While transcription factors were the only class of regulatory factors considered here, our results do not exclude the likelihood that additional factors, such as post-transcriptional RNA-binding proteins [47] or post-translational factors (kinases, methyltransferases, chromatin modifying enzymes, and so on) $[48,49]$, also contribute to the timing control of modular gene expression.

\section{Genes with complex heterochrony associate with multiple timing patterns}

While we found 1,828 genes that strongly associate within individual timing modules (module-specific genes), another 1,887 genes (31\%) instead show strong associations across timing modules (between-module genes); these between-module genes may exhibit a complex pattern of heterochrony. Our hypothesis of modular timing control suggests that negative pleiotropic effects due to changes in control may be minimized for genes with complex heterochrony by combinatorial regulation, using TFs with different timing patterns rather than the same timing pattern. First, we found no TF that significantly associates with the 1,887 genes with complex heterochrony compared to module-specific genes (FDR < 0.1). We also evaluated whether the number of module-specific TFs regulating a gene with complex heterochrony correlates with the number of timing modules represented by these TFs and obtained a rank correlation of $0.71\left(P<10^{-10}\right)$. While some correlation is expected by chance, we found only three genes (Erg11, Sis1, and YMR196W) that are strictly regulated by multiple TFs from the same timing module (three TFs for each), suggesting that this type of regulation may be rare. Thus, genes that associate with multiple timing modules tend to be regulated by multiple different timing patterns. This suggests that complex patterns of heterochronic divergence could be generated combinatorially while minimizing negative pleiotropic effects.

\section{Discussion}

Transcriptome divergence in the yeast cell-division cycle is highly time-dependent. While within-species divergence in genome-wide gene expression levels is consistent with strong stabilizing selection at each timepoint of the celldivision cycle, a large fraction of genes show significant divergence in their dynamical patterns of expression. In addition, the magnitude, direction, and degrees of freedom of transcriptome covariation change across the cell-division cycle, concordant with time-specific changes in regulatory complexity. While we could not test explicitly for the evolutionary mode of expression dynamics, we found that the major directions of within-species covariation associate with specific functional categories at different timepoints but not with neutrally-evolving genes; these directions do not predict the direction of between-species divergence for our outgroup S. paradoxus; and the S. cerevisiae laboratory strain shows extensive divergence in expression dynamics, comparable to $S$. paradoxus. These results suggest considerable potential for non-neutral evolution of expression dynamics, despite strong stabilizing selection on mean expression levels.

Since widespread divergence in transcriptome dynamics might be explained by extensive qualitative changes in gene coregulation, we assessed the similarity of gene coexpression structure across strains. Consistent with this possibility, we found significant divergence in genome-wide and modular coexpression structure, across the entire cell-division cycle and in a time-dependent manner. However, divergence in temporal coexpression does not assure divergence in coregulation; two genes may be coregulated yet exhibit distinct temporal expression trajectories (or vice-versa, for example, Figure 6B). Therefore we evaluated the possibility of heterochronic divergence, relating genes by shared changes in expression timing, rather than by similarity of expression levels (that is, coexpression). The majority of genes show timing changes consistent with heterochronic divergence, suggesting that evolution of the yeast CDC-transcriptome may be characterized as predominantly heterochronic rather than heterometric.

Genome-wide heterochronic divergence implies changes in the control of genome-wide timing patterns. However, changes in timing control (just like changes in coregulation) are expected to have negative pleiotropic consequences in natural populations, such as our yeast strains, given a global, cascading regulatory architecture. We hypothesized that negative pleiotropic effects could be minimized if regulatory architecture is instead organized into distinct timing modules which could exhibit different timing patterns. In support of this hypothesis, we found significant modularity in the genome-wide patterns of heterochrony, evidence supporting the coherence of timing modules as functionally integrated units, and dozens of transcription factors that are significantly associated with controlling these timing modules. Thus, widespread divergence in yeast transcriptome 
dynamics may be explained by heterochronic divergence in the temporal expression patterns of module-specific regulatory factors that in turn affect the timing of downstream gene expression events. Our results suggest that the short-term evolution of yeast regulatory architecture may entail preferentially quantitative changes in regulation, consistent with the established relationship between trans regulatory variation and expression divergence within species [9-13] and conservation of transcription factor binding patterns between species [22]. Although our evidence supports the role of transcription factors specifically in driving heterochronic divergence, additional factors that regulate either the production or degradation of mRNA transcripts are likely to play a significant role. Future studies incorporating additional yeast strains or higher resolution time series data may facilitate identification of additional module-specific regulatory factors and help to reveal the fine-scale structure of timing control in the yeast cell-division cycle.

\section{Conclusions}

Our data suggest a new view of molecular cell processes as a collection of dynamically-autonomous event timelines whose modularity allows divergence in gene regulation, while alleviating system-wide negative effects of regulatory change. Control of gene expression may utilize a general architecture comprised of multiple discrete event timelines that serve as a basis set of timing patterns. Interactions among module-specific regulatory factors may determine individual event timelines, and superposition different timelines may generate combinatorial complexity in regulatory patterns. This modular dynamical architecture may facilitate the generation of complex regulatory variation via changes in the scheduling and coordination of discrete event timelines, while buffering variation in individual gene expression. In this way, the architecture of genomewide timing control may bias a population's evolutionary dynamics.

\section{Materials and methods Yeast strains}

The ten natural S. cerevisiae and S. paradoxus strains are heterothallic haploid MATa derivatives of homothallic diploids. Woodland isolates were previously collected from state parks in Pennsylvania and New Jersey, USA [50] (Table S1 in Additional file 1). Laboratory strain YPS183 (HOL:kanMX, leu2A) derives from BY4741. Mating-type switching was prevented by homologous recombination of a Kanamycin resistance cassette at the HO endonuclease locus (YDL227C). The 23 mutation accumulation lines (provided by C. Zeyl [51]) are diploid and were propagated asexually for 600 generations from a Y55 ancestor (leu2 $\Delta)$.

\section{Synchronization and sampling of yeast cultures}

Strains were inoculated from frozen stock and cultured overnight in synthetic dextrose (SD) minimal medium at $30^{\circ} \mathrm{C}(225 \mathrm{rpm})$. The next day cultures were diluted into fresh SD and upon reaching a culture density of $O D \approx$ $0.25, \alpha$-factor mating pheromone was added to a final concentration of $4 \mu \mathrm{M}$. Cultures were then incubated approximately $75 \mathrm{~min}$. until arrested and synchronized in late $G_{1}$. The state of synchronization was determined by the appearance of $<10 \%$ shmoos and $<10 \%$ budding cells, visualized by light microscopy $(100 \times$, oil). Cultures were released from arrest by removing $\alpha$-factor: $2 \times$ wash with $4^{\circ} \mathrm{C} \mathrm{S}$ medium (SD without dextrose) and resuspension of cell pellets with fresh $18^{\circ} \mathrm{C}$ SD medium. Approximately $25 \mathrm{ml}$ aliquots of each culture were distributed into 18 flasks and incubated at $18^{\circ} \mathrm{C}(225 \mathrm{rpm})$. Incubation of cultures at $18^{\circ} \mathrm{C}$ in $\mathrm{SD}$ medium more than doubles the CDC-period, allowing a more accurate comparison of measurements across strains by reducing temporal sampling variation.

The sampling time course consisted of 18 samples, taken at average intervals of $19 \mathrm{~min}$. (real time), starting at $0 \mathrm{~min}$. (time of release from arrest) and ending at 345 min. The first sample $(0 \mathrm{~min}$.) was taken after all flasks were returned to the incubator. Upon sampling, each culture was placed on dry ice, mixed with $20 \mathrm{ml}$ of $-20^{\circ}$ $\mathrm{C} 100 \% \mathrm{EtOH}$ in a $50 \mathrm{ml}$ Falcon tube, inverted, and placed immediately into a $-80^{\circ} \mathrm{C}$ freezer.

\section{Microarray processing and analysis}

Total RNA was extracted from each frozen cell culture sample using Qiagen's RNeasy Kit, following manufacturer's instructions. cDNA was prepared from $15 \mu \mathrm{g}$ of each RNA sample using SuperScript III reverse transcriptase (Invitrogen) and compared directly to unsynchronized S. cerevisiae cDNA (YPS183 cultured at $30^{\circ} \mathrm{C}$ in YPD until reaching $\left.O D_{600} 1.1\right)$ on 2-channel spottedoligo glass microarrays in a common reference design. Invitrogen AlexaFluor 555 and 647 fluorophores were used to label each cDNA sample. Hybridized slides were incubated for 24-65 hours at $42^{\circ} \mathrm{C}$. Slides were prepared for scanning by serial incubation in wash buffers and dried using both a vacuum and high-purity, filtered $\mathrm{N}_{2}$ gas.

Samples were hybridized to two dye-swapped microarrays. Unsynchronized MA line transcriptomes were produced with the same design. Corning UltraGAPS glass slides, spotted with the Operon AROS for Saccharomyces cerevisiae, V1.1, were used for all hybridizations. Each microarray targets 6388 protein-coding genes using two replicate spots per oligo, yielding four technical expression measurements per gene, strain, and timepoint. In total 378 time-series and 45 unsynchronized microarrays were produced for natural and MA lines, 
respectively. Data were quantified, filtered, and normalized, yielding expression measurements for 5879.9 genes per strain on average (92.4\%). Measurements show a grand mean standard error (SE) of 0.175 . Using two microarrays of the same strain independently cultured, synchronized, and sampled at $63 \mathrm{~min}$., biological replicate measurement error was estimated as 0.554 (SE). Microarray data are available from the NCBI GEO database under accession number [GEO:GSE24237] and from the authors' web site [52].

A set of 91 transposable (Ty) element genes were excluded from the final data collection. The remaining 6,263 gene expression trajectories were imputed for missing data and calibrated to a common CDC-period of $267 \mathrm{~min}$. using budding index measurements. A common set of 6,082 genes have CDC expression for all ten natural strains. Custom software written in Python, $R$, SAS, and Mathematica was used to carry out computational analyses as described in the Supplemental Materials and methods.

\section{Additional material}

Additional file 1: Supplemental materials and methods; text, figures, and tables. This file contains 10 texts, 33 figures, and 12 tables. Additional file 2: Yeast heterochronic network. This spreadsheet details the 5,393 significant gene-gene heterochronic interactions, 1,828 module-specific genes, and 1,887 genes with complex heterochrony.

\section{Abbreviations}

CDC: cell-division cycle; FDR: false discovery rate; FWER: family-wise error rate; LDA: linear discriminant analysis; LFA: latent factor analysis; MA: mutation accumulation; PCA: principal component analysis; RMSE: root mean squared error; SD: synthetic dextrose; SE: standard error; SVD: singular value decomposition; TF: transcription factor.

\section{Acknowledgements}

We wish to acknowledge H. Murphy, C. Winter, F. Ge, E. Daugharthy, A. Goodman, and I. Gawlas for assistance, as well as M. Lee, P. Shah, and two anonymous reviewers for constructive criticism on the manuscript. This work is supported in part by a HRFF grant to the University of Pennsylvania from the Common Wealth of Pennsylvania and a NRSA Training Grant in Computational Genomics from the University of Pennsylvania (DFS). The funding bodies had no role in study design; in collection, analysis, or interpretation of data; in the writing of the manuscript; or in the decision to submit the manuscript for publication

\section{Author details}

'Department of Biology, University of Pennsylvania, 433 S. University Ave., Philadelphia, PA 19104, USA. ²Penn Genome Frontiers Institute, 433 S. University Ave., Philadelphia, PA 19104, USA.

\section{Authors' contributions}

JK and DFS designed experiments in consultation with PDS. CF performed genetic transformations of woodland yeast strains, which were isolated by PDS. DFS collected RNA and generated expression data. DFS and JK developed computational analyses, and DFS carried them out. DFS and JK wrote the paper. All authors read and approved the final manuscript.

Received: 27 May 2010 Revised: 30 August 2010 Accepted: 22 October 2010 Published: 22 October 2010
References

1. Rifkin SA, Kim J, White KP: Evolution of gene expression in the Drosophila melanogaster subgroup. Nat Genet 2003, 33:138-144.

2. Rifkin SA, Houle D, Kim J, White KP: A mutation accumulation assay reveals a broad capacity for rapid evolution of gene expression. Nature 2005, 438:220-223

3. Denver DR, Morris K, Streelman JT, Kim SK, Lynch M, Thomas WK: The transcriptional consequences of mutation and natural selection in Caenorhabditis elegans. Nat Genet 2005, 37:544-548.

4. Simon I, Barnett J, Hannett N, Harbison CT, Rinaldi NJ, Volkert TL, Wyrick JJ, Zeitlinger J, Gifford DK, Jaakkola TS, Young RA: Serial regulation of transcriptional regulators in the yeast cell cycle. Cell 2001, 106:697-708.

5. Lee TI, Rinaldi NJ, Robert F, Odom DT, Bar-Joseph Z, Gerber GK, Hannett NM, Harbison CT, Thompson CM, Simon I, Zeitlinger J, Jennings EG, Murray HL, Gordon DB, Ren B, Wyrick JJ, Tagne JB, Volkert TL, Fraenkel E, Gifford DK, Young RA: Transcriptional regulatory networks in Saccharomyces cerevisiae. Science 2002, 298:799-804.

6. Harbison CT, Gordon DB, Lee TI, Rinaldi NJ, Macisaac KD, Danford TW, Hannett NM, Tagne JB, Reynolds DB, Yoo J, Jennings EG, Zeitlinger J, Pokholok DK, Kellis M, Rolfe PA, Takusagawa KT, Lander ES, Gifford DK, Fraenkel E, Young RA: Transcriptional regulatory code of a eukaryotic genome. Nature 2004, 431:99-104.

7. Luscombe NM, Babu MM, Yu H, Snyder M, Teichmann SA, Gerstein M: Genomic analysis of regulatory network dynamics reveals large topological changes. Nature 2004, 431:308-312.

8. Stearns SC, Magwene P: The naturalist in a world of genomics. Am Nat 2003, 161:171-180.

9. Wittkopp PJ, Haerum BK, Clark AG: Evolutionary changes in cis and trans gene regulation. Nature 2004, 430:85-88.

10. Borneman AR, Gianoulis TA, Zhang ZD, Yu H, Rozowsky J, Seringhaus MR, Wang LY, Gerstein M, Snyder M: Divergence of transcription factor binding sites across related yeast species. Science 2007, 317:815-819.

11. Wittkopp PJ, Haerum BK, Clark AG: Regulatory changes underlying expression differences within and between Drosophila species. Nat Genet 2008, 40:346-350.

12. Tirosh I, Reikhav S, Levy AA, Barkai N: A yeast hybrid provides insight into the evolution of gene expression regulation. Science 2009, 324:659-662.

13. McManus CJ, Coolon JD, Du MO, Eipper-Mains J, Graveley BR, Wittkopp PJ: Regulatory divergence in Drosophila revealed by mRNA-seq. Genome Res 2010, 20:816-825.

14. Yvert G, Brem RB, Whittle J, Akey JM, Foss E, Smith EN, Mackelprang R, Kruglyak $L$ : Trans-acting regulatory variation in Saccharomyces cerevisiae and the role of transcription factors. Nat Genet 2003, 35:57-64.

15. Wang D, Sung HM, Wang TY, Huang CJ, Yang P, Chang T, Wang YC, Tseng DL, Wu JP, Lee TC, Shih MC, Li WH: Expression evolution in yeast genes of single-input modules is mainly due to changes in trans-acting factors. Genome Res 2007, 17:1161-1169.

16. Chang YW, Robert Liu FG, Yu N, Sung HM, Yang P, Wang D, Huang CJ, Shih MC, Li WH: Roles of cis-and trans-changes in the regulatory evolution of genes in the gluconeogenic pathway in yeast. Mol Biol Evol 2008, 25:1863-1875.

17. Sung HM, Wang TY, Wang D, Huang YS, Wu JP, Tsai HK, Tzeng J, Huang CJ, Lee YC, Yang P, Hsu J, Chang T, Cho CY, Weng LC, Lee TC, Chang TH, Li WH, Shih MC: Roles of trans and cis variation in yeast intraspecies evolution of gene expression. Mol Biol Evol 2009, 26:2533-2538.

18. Emerson JJ, Hsieh LC, Sung HM, Wang TY, Huang CJ, Lu HHS, Lu MYJ, Wu SH, Li WH: Natural selection on cis and trans regulation in yeasts. Genome Res 2010, 20:826-836.

19. Han JS, Szak ST, Boeke JD: Transcriptional disruption by the L1 retrotransposon and implications for mammalian transcriptomes. Nature 2004, 429:268-274.

20. Stranger BE, Forrest MS, Dunning M, Ingle CE, Beazley C, Thorne N, Redon R, Bird CP, de Grassi A, Lee C, Tyler-Smith C, Carter N, Scherer SW, Tavaré S, Deloukas $P$, Hurles ME, Dermitzakis ET: Relative impact of nucleotide and copy number variation on gene expression phenotypes. Science 2007, 315:848-853.

21. De S, Teichmann SA, Babu MM: The impact of genomic neighborhood on the evolution of human and chimpanzee transcriptome. Genome Res 2009, 19:785-794.

22. Bradley RK, Li XY, Trapnell C, Davidson S, Pachter L, Chu HC, Tonkin LA, Biggin MD, Eisen MB: Binding site turnover produces pervasive 
quantitative changes in transcription factor binding between closely related Drosophila species. PLoS Biol 2010, 8:e1000343.

23. Beer MA, Tavazoie $\mathrm{S}$ : Predicting gene expression from sequence. Cell 2004, 117:185-198

24. Yuan Y, Guo L, Shen L, Liu JS: Predicting gene expression from sequence: a reexamination. PLoS Comput Biol 2007, 3:e243.

25. Prill RJ, Iglesias PA, Levchenko A: Dynamic properties of network motifs contribute to biological network organization. PLoS Biol 2005, 3:e343.

26. Alexander RP, Kim PM, Emonet T, Gerstein MB: Understanding modularity in molecular networks requires dynamics. Sci Signal 2009, 2:pe44.

27. Kim J, Kerr JQ, Min GS: Molecular heterochrony in the early development of Drosophila. Proc Natl Acad Sci USA 2000, 97:212-216.

28. Somel M, Franz H, Yan Z, Lorenc A, Guo S, Giger T, Kelso J, Nickel B, Dannemann M, Bahn S, Webster MJ, Weickert CS, Lachmann M, Paabo S, Khaitovich P: Transcriptional neoteny in the human brain. Proc Natl Acad Sci USA 2009, 106:5743-5748.

29. Olson ME, Rosell JA: Using heterochrony to detect modularity in the evolution of stem diversity in the plant family Moringaceae. Evolution 2006, 60:724-734.

30. Moss EG: Heterochronic genes and the nature of developmental time. Curr Biol 2007, 17:R425-34.

31. Landry CR, Lemos B, Rifkin SA, Dickinson WJ, Hartl DL: Genetic properties influencing the evolvability of gene expression. Science 2007 317:118-121.

32. Gu Z, David L, Petrov D, Jones T, Davis RW, Steinmetz LM: Elevated evolutionary rates in the laboratory strain of Saccharomyces cerevisiae. Proc Natl Acad Sci USA 2005, 102:1092-1097.

33. Rifkin SA, Atteson K, Kim J: Constraint structure analysis of gene expression. Funct Integr Genomics 2000, 1:174-185.

34. Phillips PC, Arnold SJ: Hierarchical comparison of genetic variancecovariance matrices. I. Using the Flury hierarchy. Evolution 1999, 53:1506-1515.

35. Schluter D: Adaptive radiation along genetic lines of least resistance. Evolution 1996, 50:1766-1774

36. Csete ME, Doyle JC: Reverse engineering of biological complexity. Science 2002, 295:1664-1669.

37. Gould SJ: Ontogeny and Phylogeny Cambridge, MA: Harvard University Press; 1977.

38. Alberch P, Gould SJ, Oster GF, Wake DB: Size and shape in ontogeny and phylogeny. Paleobiology 1979, 5:296-317.

39. Bonner JT: Size and Cycle: An Essay on the Structure of Biology Princeton, NJ: Princeton University Press; 1965.

40. Winzeler EA, Shoemaker DD, Astromoff A, Liang H, Anderson $K$, Andre B, Bangham R, Benito R, Boeke JD, Bussey H, Chu AM, Connelly C, Davis K, Dietrich F, Dow SW, El Bakkoury M, Foury F, Friend SH, Gentalen E, Giaever G, Hegemann JH, Jones T, Laub M, Liao H, Liebundguth N, Lockhart DJ, Lucau-Danila A, Lussier M, M'Rabet N, Menard P, Mittmann M, Pai C, Rebischung C, Revuelta JL, Riles L, Roberts CJ, Ross-MacDonald P, Scherens B, Snyder M, Sookhai-Mahadeo S, Storms RK, Véronneau S, Voet M, Volckaert G, Ward TR, Wysocki R, Yen GS, Yu K, Zimmermann K, Philippsen P, Johnston M, Davis RW: Functional characterization of the $S$. cerevisiae genome by gene deletion and parallel analysis. Science 1999, 285:901-906.

41. Hughes TR, Marton MJ, Jones AR, Roberts CJ, Stoughton R, Armour CD, Bennett HA, Coffey E, Dai H, He YD, Kidd MJ, King AM, Meyer MR, Slade D, Lum PY, Stepaniants SB, Shoemaker DD, Gachotte D, Chakraburtty K, Simon J, Bard M, Friend SH: Functional discovery via a compendium of expression profiles. Cell 2000, 102:109-126.

42. Li F, Long T, Lu Y, Ouyang Q, Tang C: The yeast cell-cycle network is robustly designed. Proc Natl Acad Sci USA 2004, 101:4781-4786.

43. Wagner GP, Altenberg L: Perspective: complex adaptations and the evolution of evolvability. Evolution 1996, 50:967-976.

44. Willmore KE, Young NM, Richtsmeier JT: Phenotypic variability: its components, measurement and underlying developmental processes. Evol Biol 2007, 34:99-120.

45. Landry CR: Systems biology spins off a new model for the study of canalization. Trends Ecol Evol 2009, 24:63-66.

46. Maclsaac KD, Wang T, Gordon DB, Gifford DK, Stormo GD, Fraenkel E: An improved map of conserved regulatory sites for Saccharomyces cerevisiae. BMC Bioinformatics 2006, 7:113.
47. Amorim MJ, Cotobal C, Duncan C, Mata J: Global coordination of transcriptional control and mRNA decay during cellular differentiation. Mol Syst Biol 2010, 6:380.

48. Jensen $\amalg$, Jensen TS, de Lichtenberg U, Brunak S, Bork P: Co-evolution of transcriptional and post-translational cell-cycle regulation. Nature 2006, 443:594-597.

49. Choi JK, Kim YJ: Epigenetic regulation and the variability of gene expression. Nat Genet 2008, 40:141-7.

50. Sniegowski PD, Dombrowski PG, Fingerman E: Saccharomyces cerevisiae and Saccharomyces paradoxus coexist in a natural woodland site in North America and display different levels of reproductive isolation from European conspecifics. FEMS Yeast Res 2002, 1:299-306.

51. Zeyl C, DeVisser JA: Estimates of the rate and distribution of fitness effects of spontaneous mutation in Saccharomyces cerevisiae. Genetics 2001, 157:53-61.

52. Comparative Yeast Time-Series Gene Expression. [http://kim.bio.upenn. edu/software/yeast-cdc.shtml].

doi:10.1186/gb-2010-11-10-r105

Cite this article as: Simola et al:: Heterochronic evolution reveals modular timing changes in budding yeast transcriptomes. Genome Biology 2010 11:R105.

\section{Submit your next manuscript to BioMed Central and take full advantage of:}

- Convenient online submission

- Thorough peer review

- No space constraints or color figure charges

- Immediate publication on acceptance

- Inclusion in PubMed, CAS, Scopus and Google Scholar

- Research which is freely available for redistribution

Submit your manuscript at www.biomedcentral.com/submit
Ciomed Central 